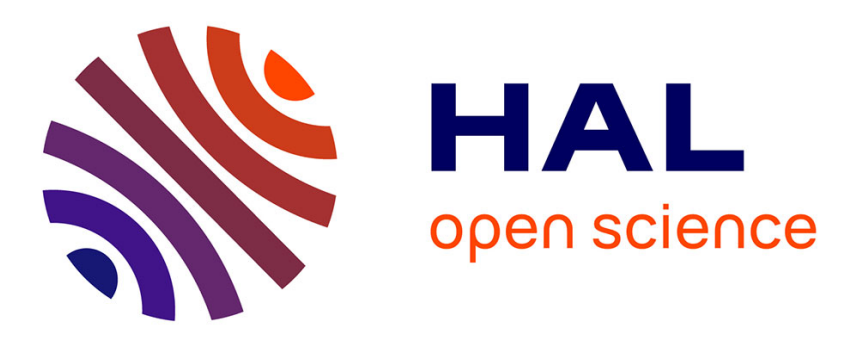

\title{
Boundary layer in pastes-Dispacement of a long object through a yield stress fluid
}

Jalila Boujlel, Mathilde Maillard, A. Lindner, Guillaume Ovarlez, Xavier

Chateau, Philippe Coussot

\section{- To cite this version:}

Jalila Boujlel, Mathilde Maillard, A. Lindner, Guillaume Ovarlez, Xavier Chateau, et al.. Boundary layer in pastes-Dispacement of a long object through a yield stress fluid. Journal of Rheology, 2012, 56 (5), pp.1083-1108. 10.1122/1.4720387 . hal-00801086

\section{HAL Id: hal-00801086 \\ https://hal-enpc.archives-ouvertes.fr/hal-00801086}

Submitted on 5 Nov 2013

HAL is a multi-disciplinary open access archive for the deposit and dissemination of scientific research documents, whether they are published or not. The documents may come from teaching and research institutions in France or abroad, or from public or private research centers.
L'archive ouverte pluridisciplinaire HAL, est destinée au dépôt et à la diffusion de documents scientifiques de niveau recherche, publiés ou non, émanant des établissements d'enseignement et de recherche français ou étrangers, des laboratoires publics ou privés. 


\title{
Boundary layer in pastes-Displacement of a long object through a yield stress fluid
}

\author{
J. Boujlel and M. Maillard \\ Laboratoire Navier (UMR ENPC-IFSTTAR-CNRS), Université Paris-Est, \\ Champs sur Marne, France
}

A. Lindner

Laboratoire PMMH, ESPCI, Paris, France

G. Ovarlez, X. Chateau, and P. Coussot ${ }^{a)}$

Laboratoire Navier (UMR ENPC-IFSTTAR-CNRS), Université Paris-Est, Champs sur Marne, France

(Received 25 December 2011; final revision received 4 May 2012;

published 19 June 2012)

\begin{abstract}
Synopsis
When it moves through a yield stress fluid, a solid object continuously reaches and liquefies new solid regions, so that both flow in liquid regions and deformations in solid regions occur. In the present work, we focus on the displacement of a plate through simple yield stress fluids (nonthixotropic). Through force vs velocity and particle imaging velocimetry measurements with a detailed analysis of the deformation history, we are able to identify the solid and liquid regions and their respective role in the flow characteristics. It is shown that the displacement of a long object through a yield stress fluid gives rise to the formation of a liquid boundary layer (BL) of uniform thickness at short distance from the leading edge, while the rest of the material remains solid. The original result is that the thickness of this boundary layer, which is of the order of $10 \mathrm{~mm}$, only slightly increases with velocity and does not tend to zero when the velocity tends to zero, in contrast with usual flows of yield stress fluids along solid surfaces. Moreover, it does not change for significant variations of the rheological characteristics of the fluid in its liquid regime. We show that these specific characteristics of the liquid layer are mainly governed by the progressive transition from an elastic solid to a liquid, starting slightly ahead of the leading edge of the plate. (C) 2012 The Society of Rheology. [http://dx.doi.org/10.1122/1.4720387]
\end{abstract}

\section{INTRODUCTION}

Various materials, such as drilling muds, fresh concrete, toothpastes, paints, foams, emulsions, are solid at rest and become liquid when submitted to a sufficiently large stress: they are yield stress fluids. The rheological behavior of these materials has been widely studied in recent years [see Coussot (2005)]. For simple (nonthixotropic) yield stress fluids such as foams, emulsions, and physical gels, a relatively good knowledge

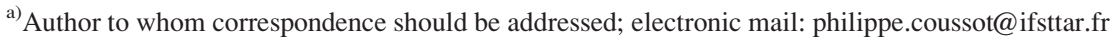


has now emerged from a mechanical point of view. This is shown by the good agreement observed between theoretical predictions from conventional (macroscopic) rheometry and local flow characteristics in simple (conduit, coaxial cylinders) or more complex geometries [Coussot et al. (2009); Ovarlez et al. (2008); Ovarlez et al. (2010); Rabideau et al. (2010)]. Here, we will not consider thixotropic materials for which the situation is more complex [Ragouilliaux et al. (2007); Ovarlez et al. (2009); Coussot and Ovarlez (2010)]. The current knowledge of the rheological behavior of simple yield stress fluids may be used in a straightforward way to describe real flows of industrial importance such as casting, pumping, coating, and spreading. In these cases, the fluid flows along solid boundaries under a given driving force.

Another class of flow problems concerns the displacement of solid objects through a yield stress fluid. These phenomena have a wide practical impact. Sedimentation of coarse particles in fresh concrete must be limited in order to avoid the development of structure heterogeneities. Drilling fluids must be able to transport rock sediments in suspension. The stability of various products, such as detergent, cosmetic, sauce, depends on the ability of bubbles, droplets, or particles of larger or smaller density than the surrounding fluid, to remain in suspension. Besides, in various processes involved in the preparation or use of pasty materials (mixing, cooking, and eating) a tool is introduced and moved through the material initially at rest in a container. This has led to the set up of a practical test for characterizing the mechanical strength of pastes which is widely used in food or building material industry, with a tool called penetrometer. It is used in a lot of standard tests such as the ASTM D217, for a variety of pasty materials such as skin creams, paints, edible fats, greases, and mortars. In this test, a solid cylinder, cone, needle, or perforated disk, is pushed under a given force through the material until stoppage. The penetration depth is considered as a "rheological" characteristic of the material. No doubt it is related to the effective rheological properties of the material but our knowledge of the flow characteristics is so far so poor that a complete relation is not yet established. At least it can reasonably be used to estimate the yield stress [Uhlherr et al. (2002); Lootens et al. (2009)]. Such a test is also used in soil mechanics or civil engineering to characterize the materials. In that case, the tool is either pushed at a constant rate or driven by repeated hammer-blows [Lowery and Morrison (2002)]. The fall cone test is also sometimes used to estimate the so-called Atterberg liquid limit of clayey soils: here this limit is defined as the water fraction allowing a cone to penetrate of $20 \mathrm{~mm}$ in $5 \mathrm{~s}$ under its own weight. At last the displacement of an object in a fluid as a result of thermal agitation is now used in microrheology for evaluating the rheological characteristics of soft-jammed systems [Habdas et al. (2004); Oppong et al. (2008); Oppong and de Bruyn (2011)]. At a larger scale, it has been used in view of determining the (macroscopic) stress tensor from the observation of bubble deformations [Dollet and Graner (2007)].

The questions to be addressed concerning the displacement of an object through a yield stress fluid are basically the force vs velocity relation and the deformation field, i.e., what is the extent of the perturbation induced by the object motion, as a function of the object shape and the rheological characteristics of the material. Actually this flow type poses a serious theoretical problem. In simple geometries (Couette, straight conduit, and open-channel flow) for which the stress distribution is given, the solid-liquid interface is fixed after some time of flow, and the distribution of the material in the liquid and solid regions does not change any more during the flow. For the displacement of an object, even a long plate, there is a change of paradigm: as the object advances, even in steady motion new solid regions are continuously reached and liquefied. This means that new deformations of the material in the solid regime continuously occur around the object and might play a significant role in the process. 
This problem has so far been mainly studied in the case of a compact object. The displacement of a sphere or a cylinder moving through a yield stress fluid has been studied in depth via theory and simulations [Ansley and Smith (1967); Beris et al. (1985); Blackery and Mistoulis (1997); Beaulne and Mitsoulis (1997); Tokpavi et al. (2008); Putz and Frigaard (2010)]. Force vs velocity expressions have been deduced and partly confirmed experimentally (although some unexplained discrepancies remain) [Atapattu et al. (1995); Jossic and Magnin (2001); Merbak et al. (2006); Tabuteau et al. (2007)]. Basically the force follows a Herschel-Bulkley (HB) type expression with an apparent shear rate equal to the ratio of the velocity to an "apparent" sheared thickness proportional to the sphere radius. Only a few works aimed at measuring directly the flow field around the object. Atapattu et al. (1995) were the first to measure the tangential velocity profiles around a sphere. Gueslin et al. (2009) provided an in-depth study of the velocity field but this concerned a thixotropic material for which there can be a further, and maybe dramatic, impact of the intense shear rate close to the object surface. Finally, Putz et al. (2008) (for spheres) and Tokpavi et al. (2009) (for cylinders) provided a new detailed data concerning the velocity field around an object moving through a yield stress fluid. They found a significant discrepancy of the velocity field with that found from simulations. In particular, they remarked an asymmetry on the flow field, the extent of the sheared zone ahead of the object being larger than expected from theory. A significant flow asymmetry was also observed in 2D foam flow around an obstacle [Dollet and Graner (2007)] which was attributed to elastic effects. We suspect that an issue in these works, which nevertheless does not necessarily explain the observed asymmetry, is the following: although they are generally considered as the flow characteristics of the fluid in the liquid regime, the measurements concern the apparent velocity field which could include both flow in the liquid regime and deformations in the solid regime. Indeed, we will see that in our case the deformations in the solid regime ahead of the object are significant.

Here, we focus on the case of a long straight object moving along its main axis through a fluid macroscopically at rest. This is an interesting alternative to compact objects as it leaves more time for observing the local flow characteristics: here, the solid region cannot close up after the passage of the object tip. We thus expect to be able to properly distinguish the solid and liquid regions.

This situation has some analogy with the classical boundary layer (BL) problem in fluid mechanics: a fluid flow around a long static object, the flow being uniform far from the object. This problem has relatively simple solutions for Newtonian fluids at large Reynolds number: the flow is localized along the plate in a thin layer of thickness increasing with the distance from the plate tip [Schlichting (1968)]. For low Reynolds number no analytical solution exists [White (1991)] but we at least know that the flow is perturbed far from the plate, typically at a distance of the order of the plate length.

On the other hand, when a plate is pushed through a simple solid material, it first induces elastic deformations which can extend far from the object [Johnson (1985)]. Then, the plate starts to penetrate the material beyond the elastic limit and plastic deformations occur. In some cases, the induced deformations are strongly localized along the plate and lead to a separation of the sample into two parts. This process is at the origin of the principle of the butter slicer. However, as far as we know there does not exist a theory describing such processes in the field of solid materials. We suggest that the flow characteristics and their dependence as a function of the detailed rheological characteristics of the material might be approached from a comprehensive description of the flow characteristics for a simple yield stress fluid.

Finally, the BL for a yield stress fluid is expected to borrow some characteristics from both the BL with simple solids and the BL with simple liquids. It should thus include 
some deformation of a solid region far from the object and a tendency to flow like a liquid around the object.

The only theoretical approaches of this problem have been developed successively by Oldroyd (1947) and Piau (2002) for a Bingham fluid and for a HB fluid [Piau and Debiane (2004)]. Piau's theory has the advantage to take into account some aspects of the impact of the deformation of the solid region on the flow characteristics in the liquid region. It predicts a BL with a lens-shape of maximum thickness increasing with the length and velocity of the plate, and with the inverse of the yield stress (see Sec. II). In particular, this theory predicts that this thickness tends toward zero when the velocity tends toward zero.

Here, we study experimentally the flow characteristics around a plate penetrating in a yield stress fluid. We find that in contrast with the existing theoretical predictions the BL is independent of the plate length and the rheological characteristics of the fluid in our experimental ranges, and slightly increases with the plate velocity. The BL finally appears to be a channel of almost uniform and constant thickness formed through the material in its solid regime, which contrasts with flows through straight conduits where the liquid region tends to disappear at vanishing velocities. More generally, by following the deformation history at a local scale our results provide for the first time a clear distinction of the solid and liquid regions in a complex flow, and a general understanding of the flow characteristics during a penetrometer test.

In the first, Sec. II we review the problem from a theoretical point of view and the predictions of existing works. Section III presents the materials and methods. In Sec. IV, we describe the general characteristics of the flow including the trajectories and deformations. An in-depth analysis of the data makes it possible to distinguish the solid and the liquid regions. In Sec. V, we focus on the velocity profiles in the liquid region and its variations as a function of the plate velocity. Section VI deals with the force vs velocity measurements and we discuss their consistency with the internal observations. On the basis of all the collected information, we present a final discussion and analysis of the problem in Sec. VII.

\section{THEORY}

\section{A. Basics}

The problem we consider here is the displacement of a two-dimensional infinite volume of a yield stress material along the direction $x$ around a thin solid plate situated at $x>0$ and $y=0$ (the plate thickness is supposed to be negligible). In practice, we can assume that far from the plate $(x \rightarrow-\infty)$, the material is pushed at a uniform velocity $V$. At least at its arrival around the plate it is somewhat deviated and sheared around the plate.

In the solid regime, we assume an elastic behavior. The solid-liquid transition is obtained when the second invariant of the deviatoric part of the stress tensor $\left(T_{I I}\right)$ is such that

$$
\sqrt{-T_{I I}}=\tau_{c}
$$

where $\tau_{c}$ is the yield stress. Note that with such a behavior the liquid regime is reached at a critical deformation which nevertheless depends on the exact initial state of the material.

In the liquid regime, we assume a HB behavior type 


$$
\Sigma=-p \mathbf{I}+\tau_{c} \frac{\mathbf{D}}{\sqrt{-D_{I I}}}+2^{n} k\left(-D_{I I}\right)^{(n-1) / 2} \mathbf{D}
$$

where $\Sigma$ and $\mathbf{D}$ are the stress and strain rate tensors, $p$ is the pressure, $\mathbf{I}$ is the unit tensor, and $k$ and $n$ are fluid parameters. The resulting behavior in simple shear is

$$
\tau<\tau_{c} \Rightarrow \dot{\gamma}=0 ; \tau>\tau_{c} \Rightarrow \tau=\tau_{c}+k \dot{\gamma}^{n},
$$

with $\tau$ and $\dot{\gamma}$ are the shear stress and shear rate amplitudes, respectively.

Note that yield stress fluids such as Carbopol solutions can exhibit some viscoelastic effects and in particular could keep, in the liquid regime, a part of the elastic energy stored in the solid regime, even if the constitutive equation is of the above form. The experimental data showing precisely the impact of elastic effects on the rheological behavior are scarce and anyway difficult to interpret around the solid-liquid transition. It is usually assumed as a first approximate that a constitutive equation like Eq. (2) accurately describes the behavior of the material in the liquid regime.

Here, for the simplicity of the presentation, we will consider the flow only along the side $y>0$, the flow characteristics on the other hand are obviously symmetrical. Far from the plate the material velocity is uniform so that the material is in its solid regime. We assume that there is no slip along the plate. This implies that for $x>0$, the velocity decreases from zero at $y=0$ to $V$ at $y \rightarrow \infty$. The material elements in contact with the plate are continuously sheared so that they necessarily become liquid after some time of flow. In contrast, far from the plate the material is negligibly deformed. As a consequence for a stationary flow there exists a given liquid region around the plate, while the rest of the material remains solid.

\section{B. The uniform BL flow}

We start by considering the (ideal) situation of a uniform $B L$ of constant thickness $\delta$ in the liquid regime and flowing along the plate. In that case, the only nonzero velocity component $(u)$ is along $x$ and only depends on the distance from the plate $y$. This is a simple shear, for which the only nonzero tangential component of the stress is $\tau_{x y}=\tau(y)$, and from Eq. (2) we find that the normal stress components of the deviatoric part of the stress tensor are equal to zero. The momentum equation is thus

$$
\begin{gathered}
0=-\frac{\partial p}{\partial x}+\frac{\partial \tau}{\partial y}, \\
0=-\frac{\partial p}{\partial y}
\end{gathered}
$$

with the boundary conditions: $u(y=0)=0$ and $u(y \rightarrow \infty)=V$. From these equations, we deduce that the pressure is constant in each plane $x=$ Cst. and that the pressure gradient along $x$ is constant. Integrating equation (4a) between 0 and $\delta$, we get

$$
\tau(y)=\tau_{w}-\left(\tau_{w}-\tau_{c}\right) \frac{y}{\delta} \text { for } 0<y<\delta,
$$

where $\tau_{w}=\tau(y=0)$ is the shear stress at the wall and

$$
\delta=-\frac{\tau_{w}-\tau_{c}}{\partial p / \partial x}
$$


For $y>\delta, \tau(y)$ is smaller than $\tau_{c}$ but undetermined. Equating the shear stress with that given by the HB constitutive equation of the material in the liquid regime (3) we find an expression for the shear rate $\dot{\gamma}=d u / d y$ which may be integrated to give the velocity profile

$$
u=V-V(1-y / \delta)^{1+1 / n} \text { for } 0<y<\delta
$$

For $y>\delta, u(y)=V$. Note that in the frame of reference linked to the fluid situated at large distance, the amplitude of the velocity is $v=V-u$. Here, the shear rate and shear stress at the wall are

$$
\begin{gathered}
\dot{\gamma}_{w}=\left(\frac{d u}{d y}\right)_{y=0}=m \frac{V}{\delta}=\frac{V}{\lambda}, \\
\tau_{w}=\tau_{c}+k \dot{\gamma}_{w}^{n}=\tau_{c}\left[1+\alpha B i^{-1}\right],
\end{gathered}
$$

in which we used $\lambda=\delta / m, m=1+1 / n$, and $\alpha=m^{n}$ and introduced the Bingham number

$$
B i=\frac{\tau_{c}}{k}\left(\frac{V}{\delta}\right)^{-n}
$$

Using the above results we find the expression for the pressure gradient as a function of the BL thickness and the velocity

$$
\frac{\partial p}{\partial x}=-\alpha \frac{\tau_{c}}{\delta} B i^{-1}
$$

This flow field corresponds to that found by Oldroyd (1947) in the case of a flow of a Bingham fluid $(n=1)$ along an infinitely long solid wall under the assumption of a pressure independent of $y$ in the liquid region. It also corresponds to that found by Piau and Debiane (2004) within the frame of his general BL theory for a HB fluid under the assumption of a BL of uniform thickness. Our analysis above shows that this result can be obtained in a straightforward way from simple assumptions.

If the pressure gradient is imposed by some external conditions, the problem is solved: the BL thickness is determined from Eq. (11) and the velocity field from Eq. (7). This is, for example, the case if there is another plate parallel to the first one and situated at a distance $2 L$. At some distance from the entrance, the flow is uniform and the above solution corresponds to the usual Poiseuille flow solution with

$$
\frac{\partial p}{\partial x}=-\frac{\tau_{c}}{L-\delta}
$$

Equating (12) with Eq. (11), we can now express $\delta$ as a function of the velocity and the rheological properties of the material in the liquid regime. For large Bingham number $(B i \gg 1)$, we have

$$
\delta \approx\left(\frac{\alpha k V^{n} L}{\tau_{c}}\right)^{1 /(1+n)}
$$


which tends to zero when $B i \rightarrow \infty$. When $B i \ll 1, \delta$ is of the order of $L$. Thus, here a BL problem in the sense of a sheared layer of small thickness compared to the characteristic length of the system is observed for sufficiently large Bingham numbers. We can also remark that the lengthscale which makes it possible to define $\delta$ is the separating distance between the plates. In the following, we will see that taking into account some specificities of the flow around a plate leads to a BL depending on other lengths.

The fundamental problem is that $\partial p / \partial x$ is a given external constant which is unknown so that $\delta$ is unknown too. When the BL thickness varies along the direction $x$, the velocity field is more complex and in particular includes a nonzero component along $y$, but the problem of the unknown pressure gradient remains the same. The existing theoretical approaches (see Sec. IIC) provided some solutions to this problem under specific assumptions, leading to explicit expressions for $\delta$ as a function of velocity and rheological parameters.

\section{BL problem}

Let us consider now the case of a liquid region $(y<\delta(x))$ of increasing thickness $\left(\delta^{\prime}(x)>0\right)$ along the plate (see example in Fig. 1). The momentum equations must now be solved in pressure and velocity which vary as a function of $x$ and $y$. We assume sufficiently slow variations of $\delta$ with $x$ so that we will describe only the main velocity component, $u$, parallel to the plate.

As for the uniform layer case, the complete solution can be found only if the pressure gradient along the solid-liquid interface is known. Oldroyd (1947) assumed a pressure varying in the form $p_{0}+2 \beta \tau_{c} \delta^{\prime}(x)$ in which $p_{0}$ and $\beta$ are two constants, and found

$$
\delta=\kappa\left(\frac{k V x}{\tau_{c}}\right)^{1 / 2},
$$

with $\kappa=3(2(1+3 \beta))^{-1 / 2}$, and

$$
\dot{\gamma}_{w}=\left(\frac{6 \beta}{1+3 \beta}\right) \frac{V}{\delta}
$$

Unfortunately, in this approach, the thickness of the BL significantly depends on the value of $\beta$, which is unknown and may significantly depend on flow and material characteristics.

In order to solve this problem Piau (2002) attempted to explicitly take into account the behavior of the material in both the solid and the liquid regions. In that aim he assumed that the stress distribution in the solid region can be found from the solution for a plate of

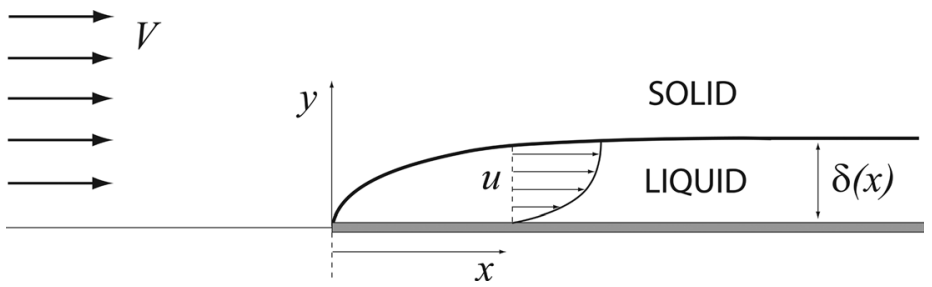

FIG. 1. Scheme of the BL problem: a uniform flow of a yield stress material at some distance from the leading edge of a solid plate; formation of a liquid region along the plate while the rest of the material remains in its solid regime. 
finite length $2 a$ immersed in an elastic medium and imposing to the fluid a shear stress equal to the yield stress along its main direction. He found that the pressure gradient may be approximated by a constant value

$$
\nabla p=-\frac{2 \tau_{c}}{\pi a}
$$

It is important to remark that here the pressure gradient, and consequently all the flow characteristics, depends on the plate length, which contrasts with Oldroyd's results [Eqs. (14) and (15)] only depending on the distance from the leading edge of the plate. It also contrasts with the pressure gradient for the uniform flow between two parallel plates only depending on the distance between the plates. This is due to the fact that the solution of the above elastic problem indeed depends on the whole surface of solid in contact with the material.

Piau (2002) for a Bingham fluid and Piau and Debiane (2004) for a HB fluid then solved the momentum equation and found that in the limit of large Bingham numbers [note that he computed this number using half the plate length $a$ instead of $\delta$ in Eq. (10)] the solution can be well approximated by a lens-shape a priori starting from the leading edge. Far from the plate edges, the flow is almost uniform, the velocity is given by Eq. (7) and the shear rate at the wall by Eq. (8), and an explicit expression for BL thickness is found by equating (16) and (11)

$$
\delta \approx\left(\frac{\alpha(\pi / 2) k V^{n} a}{\tau_{c}}\right)^{1 /(1+n)} .
$$

This approach marks a significant improvement and clearly shows that the BL problem in a yield stress fluid cannot be solved without taking into account the material behavior both in the solid and the liquid regimes. However, the basic underlying assumption of this approach is questionable: it is not clear why the pressure distribution found in the purely elastic regime (before yielding) could be used for describing the pressure distribution in a solid region coexisting with a liquid region. In particular, we can expect that as soon as a liquid region has formed in the region of largest strains, i.e., at the leading edge, there might be some significant rearrangement of the stress distribution around, which then could evolve quite differently from that found in the purely elastic regime even in the solid region. Our results below will precisely show that the characteristics of the BL shape are essentially determined around the leading edge, which means that the $\mathrm{BL}$ is in fact independent of the solution in the elastic regime for the whole plate. It is worth emphasizing that none of the above approaches explicitly describes the solid-liquid transition as it may occur around the tip when the critical deformation is reached.

\section{MATERIALS AND PROCEDURES}

\section{A. Materials}

We used solutions of Carbopol (Carbopol U10) in water, whose structure is essentially that of a "glass comprised of individual elastic microsponges" with a typical element size of 2-20 $\mu \mathrm{m}$ [Piau (2007)]. Three different concentrations (weight percent): 0.3\%, 0.4\%, and $0.5 \%$ were used. The preparation of Carbopol solution begins with a Heidolpf plastic agitator being added to a glass container filled with the appropriate amount of water and set at a rate of $1000 \mathrm{rpm}$. The appropriate amount of raw Carbopol powder was then 
slowly added to the stirring water and allowed to incorporate. Sodium hydroxide is quickly added to the solution thus increasing the $p \mathrm{H}$ of the acidic solution, while displacing the agitator throughout the entire container to ensure homogenous incorporation. For a complete homogenization, the Carbopol solution is then mixed for approximately $24 \mathrm{~h}$ in a mortar mixer.

A transparent emulsion with a rheological behavior qualitatively similar to that of the Carbopol solutions [Goyon et al. (2010)] was also prepared only for purpose of direct qualitative observations: it is made of silicon oil droplets (volume fraction: $82.5 \%$ ) in a water-glycerin mixture (50\% weight fraction) stabilized by a surfactant (trimethyl (tetradecyl) ammonium bromide $20 \mathrm{~g} / \mathrm{l}$ ). The preparation of this material starts by dissolving the surfactant in the water-glycerin mixture to obtain a homogeneous solution without lumps. Then, the oil is added gradually and dispersed in aqueous phase by mixing the system with a Silverson mixer at $600 \mathrm{rpm}$ for a first period then at $6000 \mathrm{rpm}$ for $10 \mathrm{~min}$. In order to ensure homogeneity of the final mixture, the apparatus is displaced in the container from top to bottom during mixing. Before using this preparation, it is centrifuged to get rid of air bubbles formed during mixing.

Rheological tests were performed with a Malvern Kinexus-stress-controlled rheometer equipped with two circular, rough plates (diameter, $6 \mathrm{~cm}$; waterproof sandpaper of average particle diameter $70 \mu \mathrm{m}$ ). Stress ramp tests were carried out, wherein the stress was increased logarithmically in time at a constant rate (for $3 \mathrm{~min}$ ) and then decreased logarithmically in time for the same time. Typical data of that type have already been published many times [see, for example, Coussot (2005)]. We kept the data in the decreasing ramp as reflecting the material behavior in steady state. We then took into account the heterogeneity of shear rate along the radial direction inherent in this geometry, which implies a slight correction [see Coussot (2005)] to finally deduce the effective flow curve of the material.

All these materials appeared to behave as simple yield stress fluids (without significant thixotropy) and their flow curve could be very well fitted over four decades in shear rate $\left[10^{-2} ; 10^{2} \mathrm{~s}^{-1}\right]$ by a HB model (see Fig. 2). For such materials, it was shown from magnetic resonance imaging (MRI) measurements that, in contrast with more complex yield stress fluids which can, in particular, display shear-banding at apparent shear rates up to a

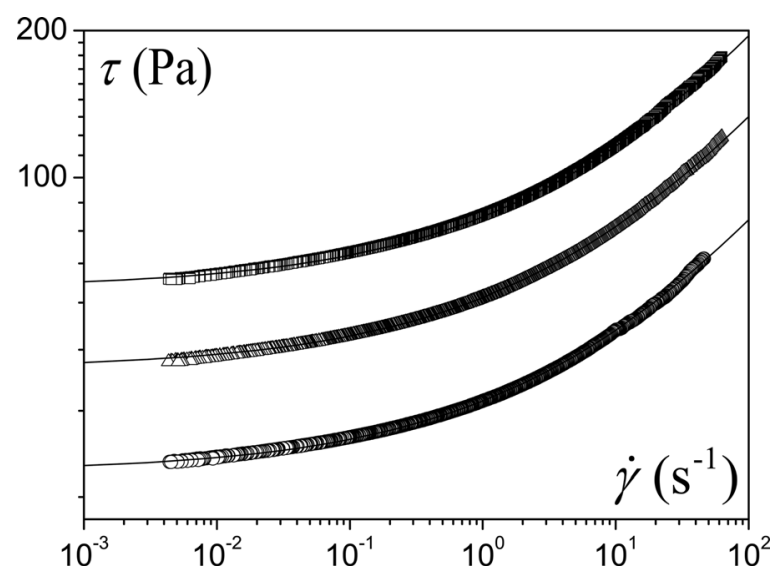

FIG. 2. Flow curves of Carbopol gels at different concentrations: (from bottom to top) $0.3 \%$; $0.4 \%$; and $0.5 \%$. The continuous lines correspond to a $\mathrm{HB}$ model [Eq. (3)] fitted to data with the following parameters

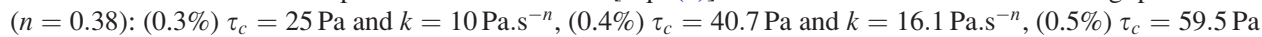
and $k=23.6 \mathrm{~Pa}_{\mathrm{s}}{ }^{-n}$. 
shear rate of the order of $10 \mathrm{~s}^{-1}$ (see Sec. I), this (HB) macroscopic constitutive equation effectively corresponds to their local rheological behavior (inside the bulk) in the range $\left[10^{-2} ; 10^{2} \mathrm{~s}^{-1}\right]$ [Coussot et al. (2009)].

In order to estimate the critical deformation separating the solid and the liquid regimes, we carried out creep tests under different stress values. After a short preshear and $20 \mathrm{~s}$ at rest the stress was imposed and we recorded the deformation in time (see Fig. 3). Then, the sample was removed, another sample was set up and the above procedure was started again for a different stress values. In that case, after some time we can rather well distinguish the solid and the liquid regimes. Below some critical stress (the yield stress), the deformation tends to saturate, it reaches a plateau, this situation corresponds to the solid regime. Note however that over long time some creep flow suggesting aging effects may be observed [see Coussot et al. (2006)]. For higher stresses, after some time the deformation increases at a constant rate, which indicates that a steady flow in the liquid regime has been reached. The critical deformation is associated with the plateau level reached for the highest stress below the yield stress. Note that for very low shear rates various effects such as some slippage or solid creep can make the analysis difficult. Moreover, it is difficult to properly control the initial stress distribution in the material, i.e., there might be some residual stresses even after a preshear and some rest. Leaving apart these problems which can hardly be controlled properly here our data suggest that the critical deformation is $0.6 \pm 0.2$ for all our Carbopol solutions.

We also carried out start up flow tests at low shear rates. In that case, the sheardependent term of the shear stress in Eq. (3) is small so that we observed a shear stress progressively increasing toward a plateau close to the yield stress (see inset of Fig. 3) and associated with a slow steady flow in the liquid regime. The deformation at which the plateau is reached is expected to be the critical deformation $\gamma_{c}$ marking the transition from the solid to the liquid regime. Once again this measurement is approximate, in particular, because we can hardly control properly the initial stress state of the material, but we again obtain a value between 0.4 and 0.8 , consistent with the value obtained from creep tests.

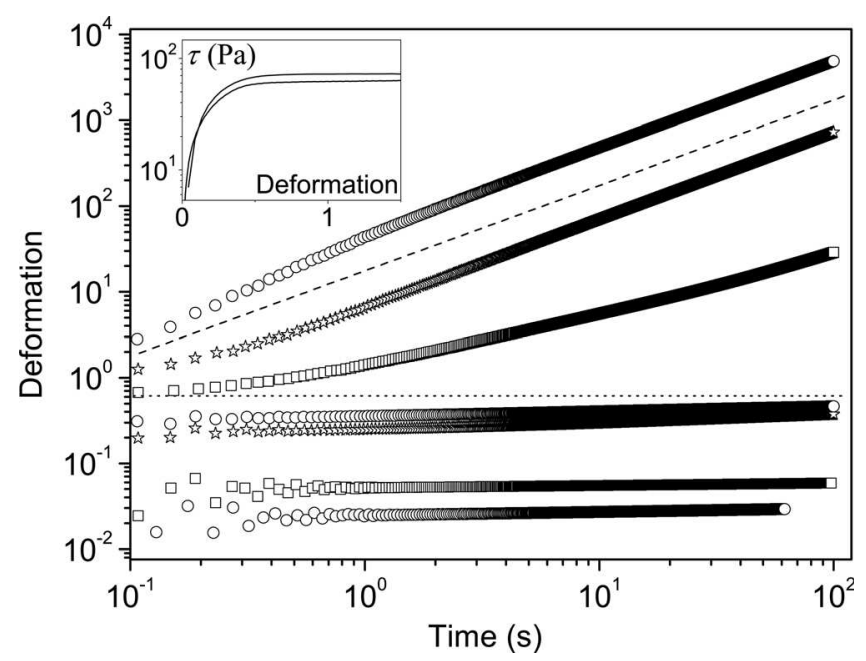

FIG. 3. Creep tests for the $0.5 \%$ Carbopol solution at different stress levels (from bottom to top): $8,15,46,54$, 77, 108, and $154 \mathrm{~Pa}$. The dashed line corresponds to a slope 1 . The dotted line corresponds to a deformation 0.6. The inset shows the stress variation as a function of deformation under constant shear rate: $0.01 \mathrm{~s}^{-1}$ (bottom), $0.1 \mathrm{~s}^{-1}$ (top). 


\section{B. Plate displacement}

We studied the BL problem by displacing vertically at a velocity $V$ a thin plate through a fluid bath in a container at rest (see Fig. 4). The vertical plate is linked to the plate of a dual-column testing system (Instron 3365) which controls the position with a resolution of $0.1 \mu \mathrm{m}$. The apparatus is equipped with a $10 \mathrm{~N}$ static load able to measure the force to within a relative value $\pm 10^{-6}$ of the maximum value. We estimated the buoyancy force from similar experiments with a Newtonian fluid at very low velocities (so that viscous effects were negligible) and a correction for the effective density of the yield stress fluid. This buoyancy force was then withdrawn from the recorded force to get the force $F$ strictly associated with the fluid resistance to the plate displacement. In our tests, the ratio of the buoyancy force per unit length to the typical viscous force per unit length (the yield stress times twice the plate width) takes value between 10 and 30\% depending on material characteristics.

Note that the question of the buoyancy expression for a yield stress is somewhat open but if we assume as usual that the constitutive equation in the solid or the liquid regime may be expressed in the form of the stress tensor function of the history of the deformation, and if the fluid is incompressible, the usual buoyancy expression $(\rho g \Omega)$ is valid. Indeed, in that case a usual pressure term can be identified in the stress tensor, and if we have found the solution for the stress tensor in the absence of gravity effects the solution with gravity effects is simply found by adding a hydrostatic pressure distribution to the solution obtained in the absence of gravity, which leads to the usual buoyancy expression.

We choose the geometry of the system in order to be as close as possible to the twodimensional flow problem considered in the theoretical part. We can expect that with a sufficiently small ratio of thickness $(e)$ to plate width $(l)$, and a small ratio of the plate thickness to the distance $((L-l) / 2)$ between the plate edges and the container sides, edge effects will be negligible. Also, we can expect that with a sufficiently small ratio of the plate thickness to the distance $(D)$ between the plate and the container, and a small ratio of the plate section $(e l)$ to the container section $(2 L D)$, the container walls will not play a significant role on the flow characteristics. We could partly check the validity of these assumptions from force measurements with various geometrical characteristics. In

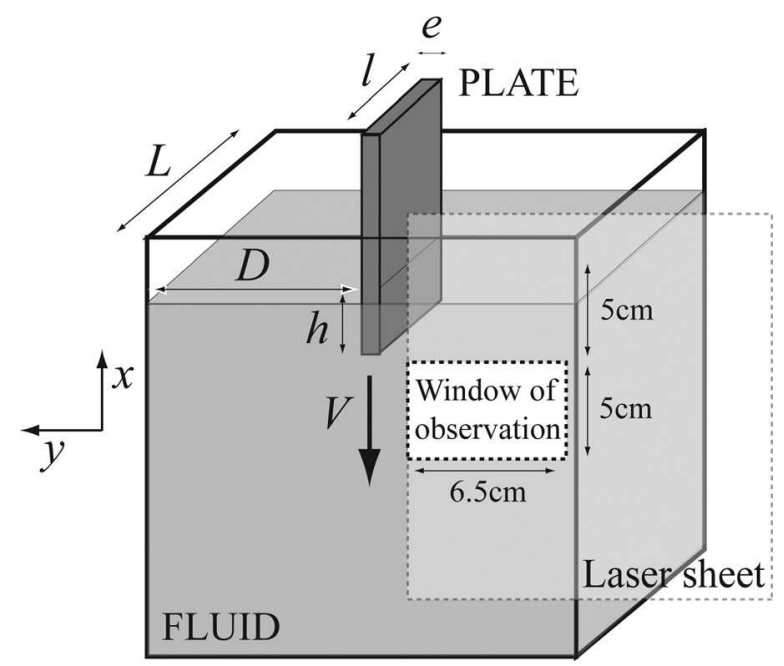

FIG. 4. Scheme of the experiment: the dark plate moves vertically through the fluid in a container. 
particular, the force does not change significantly as long as $e / D$ is smaller than $1 / 20$, and as long as $e / l$ is smaller than $1 / 25$. However, a more systematic study with the help of imaging is needed in order to know the effective impact of the geometry on the flow characteristics. In the present study, we chose to focus on the detailed flow characteristics in a specific case falling in the range for which we can consider to have a $2 \mathrm{D}$ flow: $e=1.5 \mathrm{~mm}, l=7 \mathrm{~cm}, L=10 \mathrm{~cm}$, and $D=7.5 \mathrm{~cm}$.

The material was poured in the container and left at rest for some time in order to ensure residual stress relaxation. In order to avoid wall slip effects we covered the solid surfaces of the plates with waterproof sandpaper of average particle diameter $70 \mu \mathrm{m}$, giving an effective roughness of a few tenths of microns, a thickness much larger than the elements of the materials used in this study. The plate was first coated with material then the material in excess of the roughness was removed (cleaning operation) before immersing the plate for the first test; afterward it was simply cleaned between two successive tests. All measurements were performed at room temperature $\left(23^{\circ} \mathrm{C}\right)$.

\section{Displacement measurement}

We measured the velocity profiles in Carbopol solutions with particle imaging velocimetry (PIV). Polystyrene beads (diameter: $80 \mu \mathrm{m}$ ), which served as markers were dispersed in the fluid at a mass weight fraction of $0.2 \%$. Their density was very close to that of the fluid so that sedimentation was negligible. We checked that they negligibly affected the rheological behavior of the mixture.

A fluid sheet perpendicular to the plate and crossing it along its central axis was illuminated by a continuous red laser of $20 \mathrm{~mW}$ (see Fig. 4). The particle displacements in this sheet were filmed with a CCD camera which was fixed relatively to the container. The window of observation (see Fig. 4) was $5 \mathrm{~cm} \times 6.5 \mathrm{~cm}$ (height per width) and situated $5 \mathrm{~cm}$ below the free surface. We started to take pictures when the plate tip appeared in that window and followed the fluid displacements during a further plate motion of $15 \mathrm{~cm}$.

For the data analysis, we used the commercial software DAVIs. The technique is based on the estimation of the particle velocity from a correlation analysis of two successive pictures (our maximum number of images per second was 16). Each picture is divided in correlation squares (of about $1 \mathrm{~mm}^{2}$ ) which are compared through their Fourier transform. The displacement of the peak in the resulting curve between the two successive times provides an estimate of the particle velocity in the square. Considering the significant size and low concentration of the markers, it appeared necessary to have a minimum displacement of the plate of $1 \mathrm{~mm}$ in order to get relevant data. We thus obtained a velocity field defined as $\left(v=v_{x}\left(x_{i}, y_{j}\right), v_{y}\left(x_{i}, y_{j}\right)\right)$, in which $x_{i}$ and $y_{j}$ are the coordinates of the different elementary volumes in a $1 \mathrm{~mm}^{2}$ mesh.

Actually, in order to reduce further the noise on the data for the analysis of transient effects we chose to average the results over ten successive pictures, which means that the plate has moved over a distance of $d=1 \mathrm{~cm}$ during a time $\Delta t$. We thus get a series of moving averaged velocity fields defined in the same mesh. Then, for the analysis, in order to focus on relevant data (avoiding any overlap of the periods of averaging) we kept the corresponding velocity fields observed at successive times associated with a displacement of $1 \mathrm{~cm}$. Typical velocity distributions obtained in this way are shown in Fig. 5.

A typical profile of the velocity along the plate direction as a function of the distance from the plate $(v(y))$ extracted from such data exhibits an almost perfect plateau at some distance, say, for $y>15 \mathrm{~mm}$ (see Fig. 6). The velocity of this plateau $(\Delta V)$ is negative and much smaller than the plate velocity: $-\Delta V \ll V$. In the data analyzed here $\Delta V / V=3 \pm 0.5 \%$. This slight upward motion obviously finds its origin in the downward 


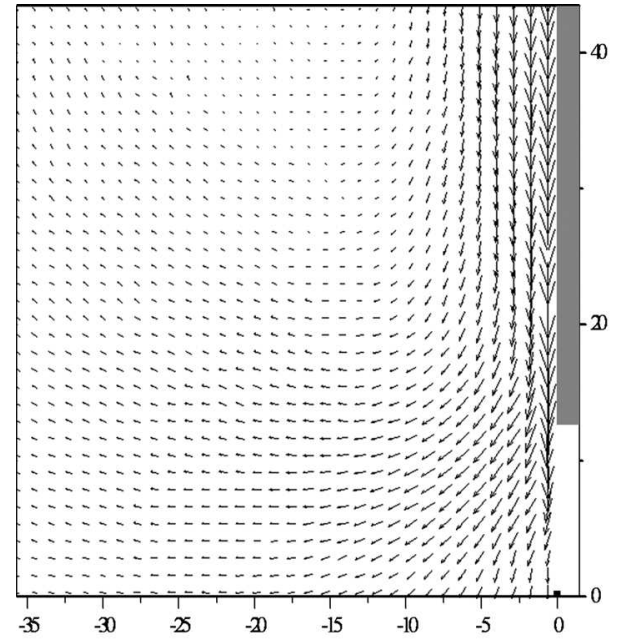

(a)

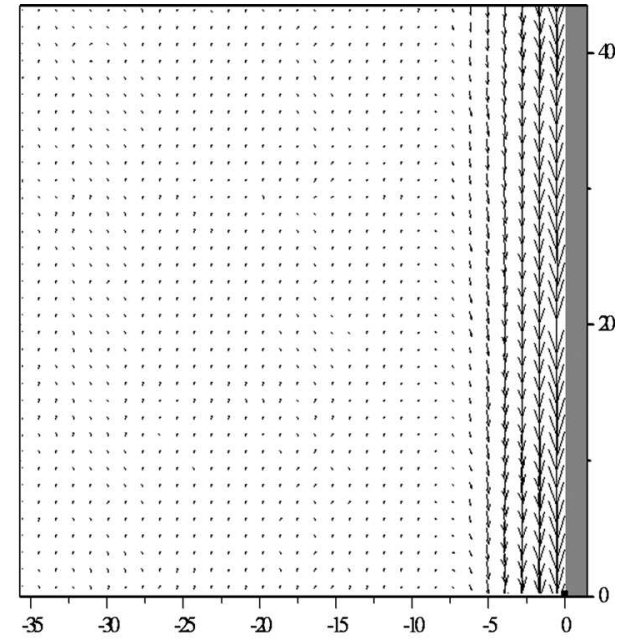

(b)

FIG. 5. Velocity field averaged over a plate displacement of $1 \mathrm{~cm}$ (Sec. III C) for $V=1 \mathrm{~mm} \cdot \mathrm{s}^{-1}$ as directly obtained from PIV measurements for two different positions of the plate with regards to the window of observation: (left) plate tip at $3 \mathrm{~cm}$ above the window bottom, (b) plate tip at $10 \mathrm{~cm}$ below the window bottom. The average plate position is represented by the grey area.

motion of the plate through the fluid and the mass conservation (assuming that the fluid is incompressible). However, the simple upward displacement of a fluid volume equal to the plate volume moving downward cannot explain such a value for $\Delta V / V$. Indeed, in that case we would expect $\Delta V / V \approx e l / 2 L D$, i.e., about $0.2 \%$. The larger value that we found results from the downward displacement of a significant volume of fluid around the moving plate. If we roughly assume that the average velocity of this (boundary) layer is half the plate velocity we find that its total thickness (including both sides of the plate) should be of

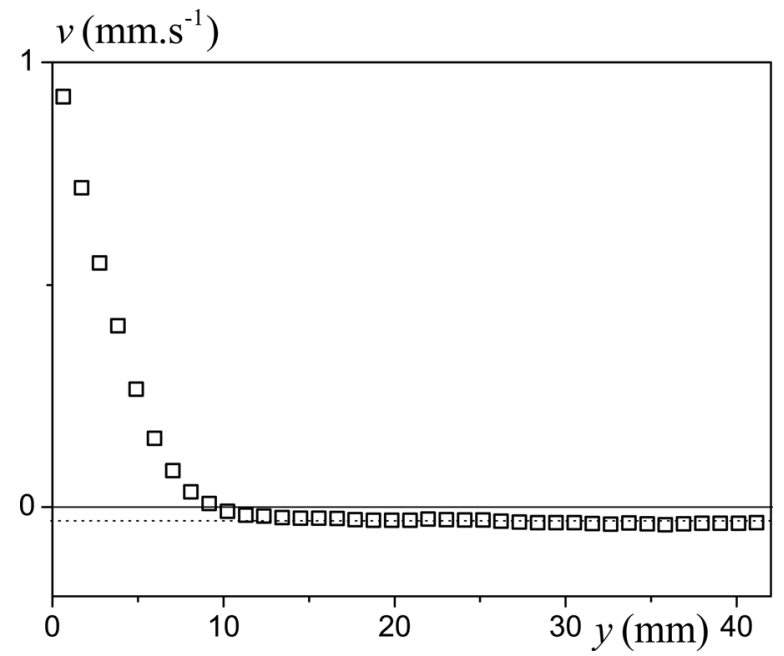

FIG. 6. Effective velocity profile (in the frame of the container) along the plate direction during the penetration through a Carbopol gel $(0.5 \%)$ at $V=1 \mathrm{~mm} / \mathrm{s}$ at $\mathrm{x}=6 \mathrm{~cm}$. The dotted line corresponds to $v=-0.03 \mathrm{~mm} / \mathrm{s}$. The plate is situated at $y=0$ and the container wall at $y=7.4 \mathrm{~cm}$. 
the order of $e^{*}=14 e \approx 25 \mathrm{~mm}$, a value which will appear quite consistent with the measurements presented below. Then, we would indeed obtain $\Delta V / V \approx e^{*} l / 2 L D \approx 3 \%$.

In the following, we will consider that the effective plate displacement relatively to the fluid occurs at $V^{*}=V+\Delta V$, slightly larger than $V$. For the consistency of the analysis in all the data presented below we shifted the velocity profiles in order to have an apparent plateau velocity equal to zero, which means that the effective relative velocity is $V^{*}$, and for the scaling by the plate velocity we used $V^{*}$. Nevertheless, for the simplicity of the presentation we will write $V$ in the text and the graphs.

\section{GENERAL ASPECTS OF THE FLOW}

\section{A. Qualitative trends}

The basic trends of the flow during the displacement of a plate through yield stress fluid are showed up from a test with a colored material in a specific configuration: here, the plate and the container had the same width (perpendicularly to the image) so that the deformation field was mostly two-dimensional. Moreover, we used a transparent emulsion colored differently (by mixing it with different inks) and successively poured the different materials to get approximately horizontal parallel layers. We took pictures (without the laser sheet) of the apparent deformation of these layers from the side of the container (see Fig. 7). The mostly 2D character of the deformation in that case is confirmed by the fact that the interfaces between these layers remain rather sharp, whereas they would likely appear diffuse for a more complex 3D shape.

From such a picture (see Fig. 7) we get a qualitative view of the strain field resulting from plate displacement through the deformations of the initial approximately straight layers. A similar picture of the strain field is obtained at any time during the plate motion through the fluid, which suggests that the flow is stationary. Beyond some distance (along $y$ ) from the plate, the shape of the interface between the different layers appears to remain almost constant, which suggests that the material is only weakly deformed. On the contrary, a region close to the plate is continuously and widely deformed as the plate advances: there the colored regions are widely stretched. This suggests that there is a flowing liquid region along the plate, while the rest of the fluid is in its solid regime.

\section{B. Flow stationarity}

Now using PIV data we start by looking at the evolution of the velocity profile at a given distance $(x)$ from the plate tip. Due to our window size and the fixed position of the

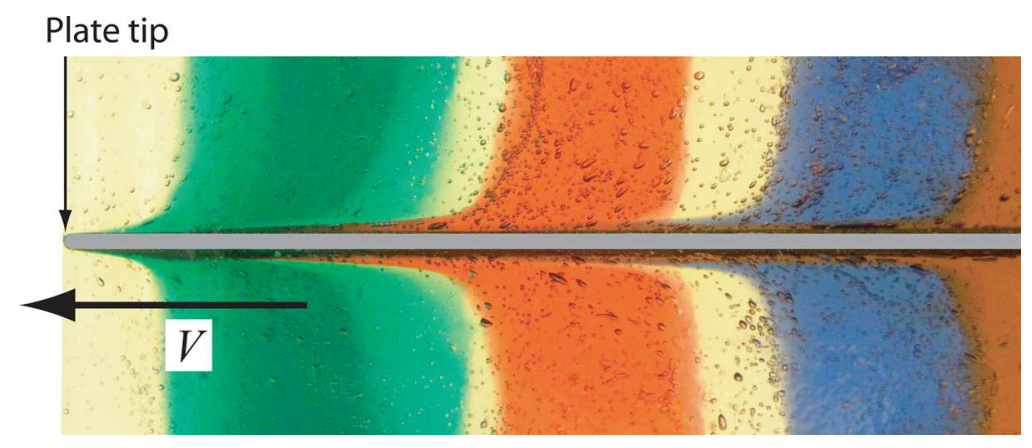

FIG. 7. Side view of the shape of colored layers of a transparent emulsion after plate penetration (Sec. IV A). The real figure height is $4.5 \mathrm{~cm}$. 


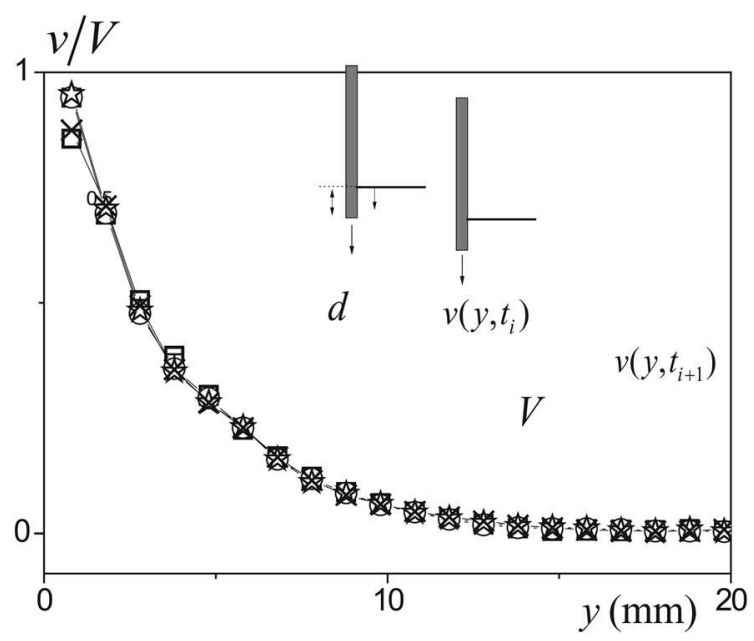

FIG. 8. Velocity profiles along the plate direction during the penetration through a Carbopol gel $(0.5 \%)$ at $V=1 \mathrm{~mm} / \mathrm{s}$ at a given distance $d$ from the plate tip $(5 \mathrm{~cm})$ and successive times $\left(t_{i}\right): 0 \mathrm{~s}$ (squares), $10 \mathrm{~s}$ (crosssquares), $20 \mathrm{~s}$ (circles), $30 \mathrm{~s}$ (stars), $40 \mathrm{~s}$ (crosses).

camera with regards to the container we were able to observe this evolution over a $5 \mathrm{~cm}$ displacement of the plate. We found that at least after a penetration of $5 \mathrm{~cm}$, for any fixed value of $x$ in the range -4 to $16 \mathrm{~cm}$, the velocity profiles over $5 \mathrm{~cm}$ of displacement of the plate were identical (see typical example of Fig. 8). This means that in our range of observation, the flow in the direction $x$ is stationary in the frame of reference of the plane.

An important consequence of this stationarity of the flow is that a single picture of the velocity field provides at the same time the spatial velocity distribution and the flow history of fluid elements in the picture.

\section{Trajectories}

On the basis of our observations concerning the flow stationarity we can use a single PIV picture of the velocity field to deduce the trajectories of fluid elements defined in $1 \mathrm{~mm}^{2}$ areas. Using the velocity of one of these fluid elements as determined by the averaging procedure described in Sec. III C, we compute the resulting displacement of this element during a plate displacement of $1 \mathrm{~cm}$. We thus reach a new position where the new velocity leads to a further displacement and so on.

The resulting trajectories in the frame of reference of the plate are shown in Fig. 9. The uneven character of some of these trajectories is likely due to the uncertainty on data. The robust observations are as follows:

(i) Around the level of the leading edge of the plate, the fluid tends to move laterally of a distance of the order of $3 \mathrm{~mm}$; this effect occurs in a similar way over several centimeters along the direction $y$.

(ii) After this lateral motion, the fluid elements tend to slightly go back toward the plate (typically of about $1 \mathrm{~mm}$ ) then roughly follow straight vertical lines; actually in Fig. 9 there is still some slight motion toward the plate at about $8 \mathrm{~cm}$ from the tip but the stabilization of the velocity profile shape beyond $8 \mathrm{~cm}$ described in Sec. V will clearly demonstrate that farther from the plate tip the trajectories are effectively parallel to the plate. 


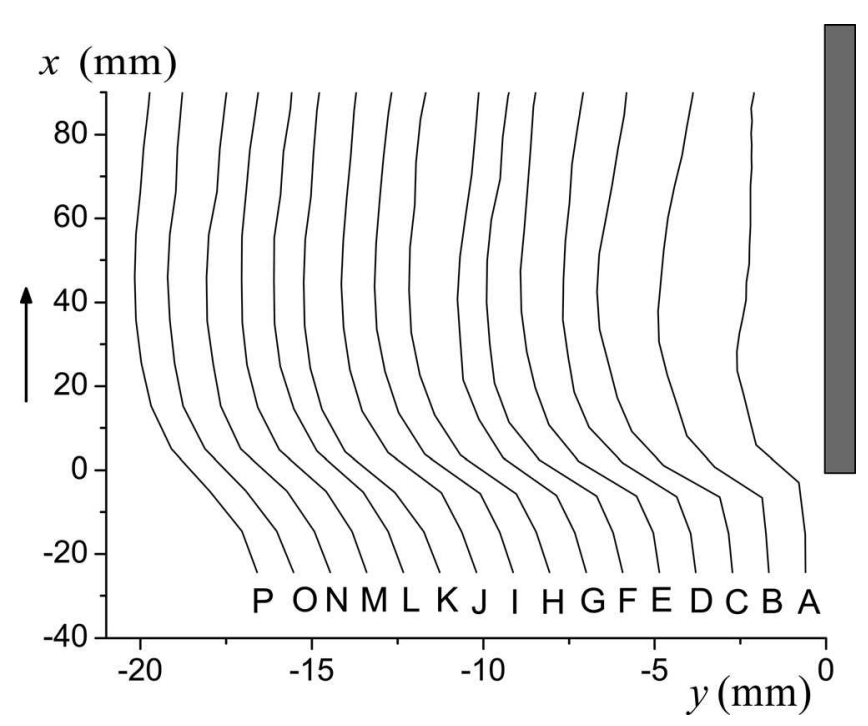

FIG. 9. Trajectories of fluid elements (named by letters from A to P) initially situated at different distances from the plate along a horizontal line at $x=-25 \mathrm{~mm}$ in the frame of reference of the plate (grey area on the right) for a penetration through a Carbopol gel $(0.5 \%)$ at $V=1 \mathrm{~mm} / \mathrm{s}$. The arrow indicates the general direction of motion of the elements along their trajectories. Note the different lengthscales used for $x$ and $y$ axes.

\section{Deformations}

We can also appreciate the deformations in the material from the above information concerning the trajectories. A typical example is shown in Fig. 10 where we follow the successive shapes and positions of an initially horizontal line of elements. The lateral displacement around the plate tip here appears from the displacement of the first point on the right of the lines. It is remarkable that the deformation of the material seems to be rather small before it has reached the level of the plate tip. Another point is that there seems to be still significant deformation (see upper lines in Fig. 10) even at several centimeters from the plate and for lines at several centimeters above the plate tip.

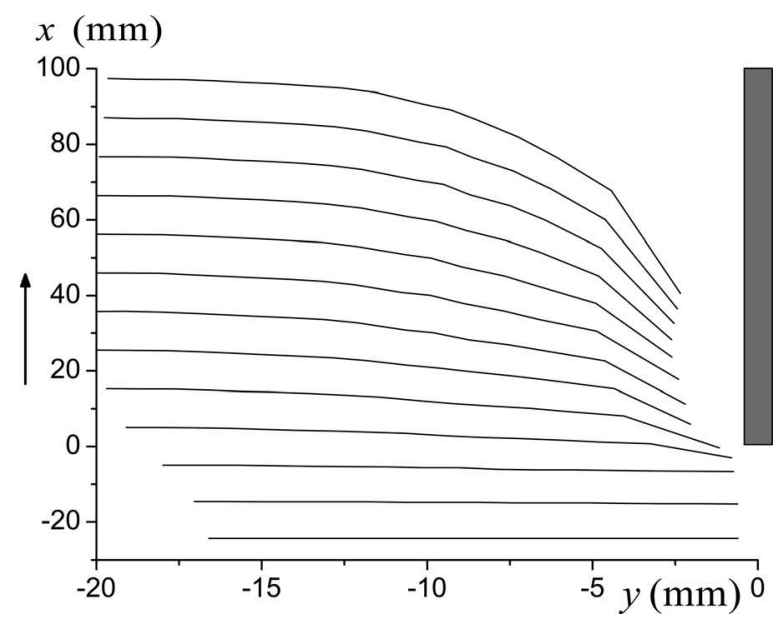

FIG. 10. Evolutions at successive times in the frame of reference of the plate of a horizontal line in the fluid initially situated at $x=-25 \mathrm{~mm}$ for the same test as described in Fig. 5: (from bottom to top) every $10 \mathrm{~s}$ from 0 to $120 \mathrm{~s}$. The arrow indicates the direction along which the line is successively deformed. 
These observations provide a global appreciation of the flow characteristics, but they do not provide any clear view of the distribution of liquid and solid regions in time. In order to address this issue we need to effectively compute the deformation of the elements along their trajectories. If the residual stresses inside the material after its preparation and set up in the container are negligible we can expect, according to Eq. (1), that a fluid element initially solid will become liquid when the total deformation it has undergone is larger than the critical deformation $\left(\gamma_{c}\right)$.

In that aim we computed the components of the $2 \mathrm{D}$ deformation gradient $\mathbf{F}$ in each elementary volume of our mesh after each incremental displacement of the plate from the following expressions of its components:

$$
\begin{aligned}
& F_{x x}\left(x_{i}, y_{j}\right)(t)=(1 / 2 d)\left[u_{x}\left(x_{i+1}, y_{j}\right)-u_{x}\left(x_{i-1}, y_{j}\right)\right]+1, \\
& F_{x y}\left(x_{i}, y_{j}\right)(t)=(1 / 2 d)\left[u_{x}\left(x_{i}, y_{j+1}\right)-u_{x}\left(x_{i}, y_{j-1}\right)\right], \\
& F_{y x}\left(x_{i}, y_{j}\right)(t)=(1 / 2 d)\left[u_{y}\left(x_{i+1}, y_{j}\right)-u_{y}\left(x_{i-1}, y_{j}\right)\right], \\
& F_{y y}\left(x_{i}, y_{j}\right)(t)=(1 / 2 d)\left[u_{y}\left(x_{i}, y_{j+1}\right)-u_{y}\left(x_{i}, y_{j-1}\right)\right]+1,
\end{aligned}
$$

in which $u_{x}=v_{x} \Delta t$ and $u_{y}=v_{y} \Delta t$ are the local displacements over the time $\Delta t$. We then computed the Green-Lagrange strain tensor e undergone by the elements along their trajectory and the corresponding second invariant of this deformation $\sqrt{-e_{I I}}$. Note that theoretically for a simple shear this invariant simply reads $\gamma / 2$. As a consequence for a steady-state simple shear this second invariant is proportional to the flow duration.

Let us follow the total deformation undergone by fluid elements initially on the horizontal line situated at $25 \mathrm{~mm}$ below the plate tip. For elements initially close to the plate, say, at a distance smaller than $7 \mathrm{~mm}$ (i.e., points A to $\mathrm{G}$ ), beyond some critical deformation the total deformation increases linearly with time: the points fall along a line of slope 1 in a logarithmic scale (see Fig. 11). This suggests that these elements are in the liquid regime and finally undergo a steady-state simple shear. On the contrary, for elements initially situated at distances larger than $8 \mathrm{~mm}$ (i.e., points $\mathrm{H}$ to $\mathrm{P}$ ) the total deformation only slightly increases beyond the critical deformation, there is almost a deformation plateau. [Note that for the fluid element initially situated at $8.1 \mathrm{~mm}$ (point $\mathrm{H}$ ) this plateau seems to develop slightly later than the other elements.] This suggests that these elements do not get out of their solid regime. A similar behavior is observed for a series of creep tests in simple shear at different shear stress values (see Sec. III A), a procedure which precisely allowed us to measure the yield stress and the critical deformation of the material.

Finally, these observations make it possible to determine the shape of the solid and the liquid regions around the plate tip. In that aim, for each trajectory of an element initially close to the plate we estimate from Fig. 11 the time at which the apparent critical deformation separating the two regimes (dashed line) has been overcome. We then consider that the liquid regime appears at the point on the trajectory (see Fig. 9) associated with this time. Thus, we get the shape of the liquid region around the plate tip (see Fig. 12). Some material is liquefied just below the tip but most of the material is liquefied around the level of the tip.

\section{VELOCITY PROFILES}

Since after $2 \mathrm{~cm}$ behind the plate tip the trajectories (see Fig. 9) are mostly parallel to the plate we can now focus at the detailed characteristics of the velocity component $(v)$ 


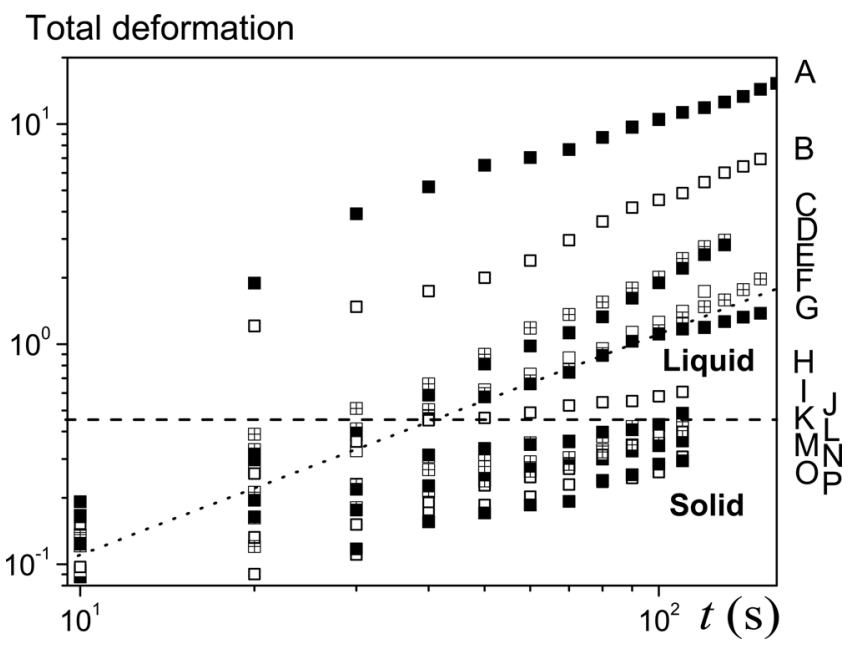

FIG. 11. Total deformation as a function of time for the elements of Fig. 9 initially along the bottom line at different distances from the plate axis: the symbols follow an alternation of filled squares, empty squares, and cross-squares, and are associated with the trajectory letters of Fig. 9. The dashed line is situated at the level of the apparent critical deformation of the material. The dotted line has a slope 1 .

along the plate axis $(x)$ as a function of time, position and plate velocity for given geometrical conditions.

\section{A. Flow profile variation from the plate tip}

Let us follow the evolution of the apparent velocity profile along the plate. Here, we look at the velocity profiles as directly measured from PIV along horizontal lines situated at different distances from the plate tip. The first velocity profiles (situated at a vertical distance from the plate tip larger than or equal to $2 \mathrm{~cm}$ ) exhibit a slight slope which is almost constant over large distance from the plate (see Fig. 13). The velocity profiles closer to or above the plate tip are made of one part along the plate in which the shear is strong and one part at larger distance in which the velocity slowly decreases. Then, the

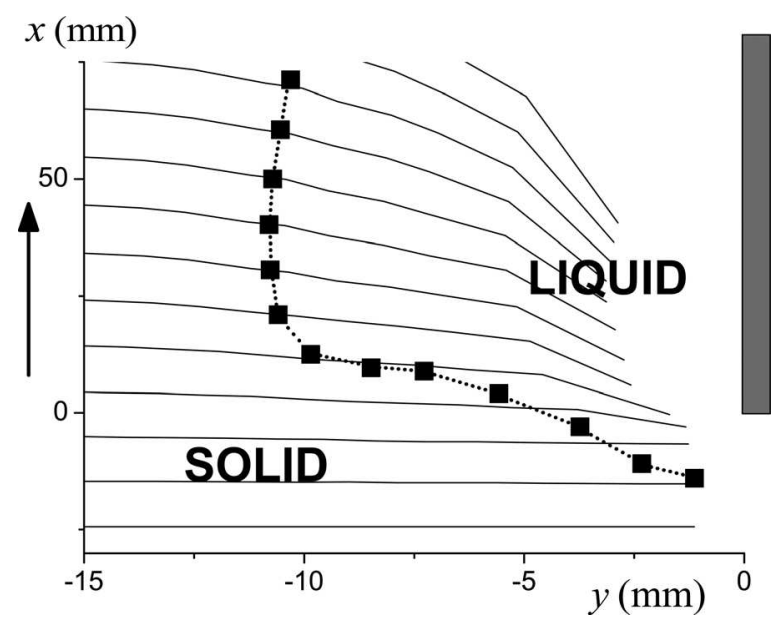

FIG. 12. Deformation of an initially horizontal line of elements (same data as in Fig. 10) and liquid-solid boundary (Sec. IV D). Note the different lengthscales used for $x$ and $y$ axes. 


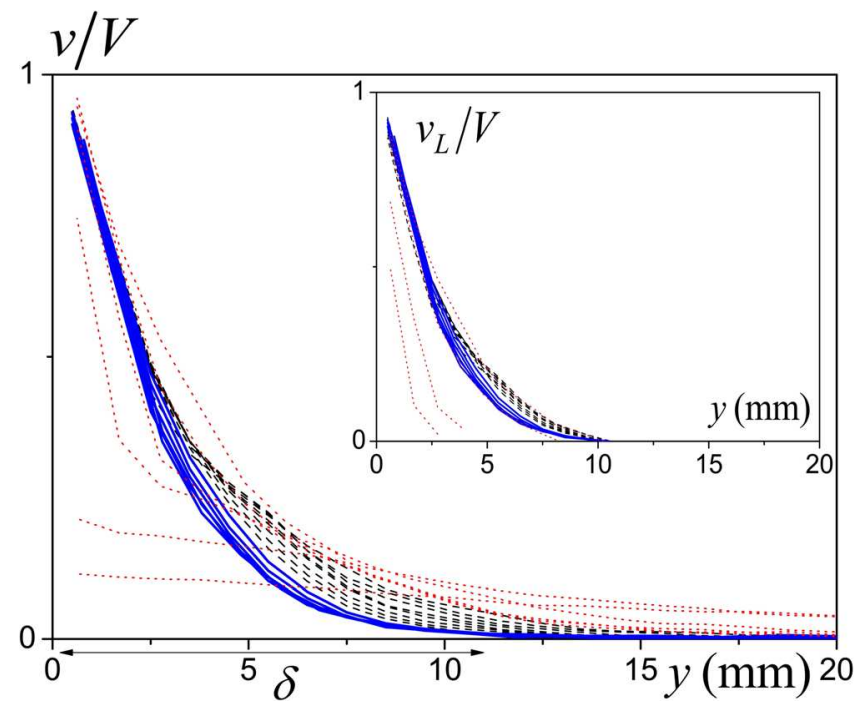

FIG. 13. Velocity profiles along horizontal lines in the material for the test presented in Fig. 5 at different distances $x$ (separated by $1 \mathrm{~cm}$ ) from the plate tip: -3 to $2 \mathrm{~cm}$ (red dotted lines), $3-11 \mathrm{~cm}$ (dashed lines), $12-17 \mathrm{~cm}$ (continuous blue lines). Inset presents the same data (except for the lines at -3 and $-2 \mathrm{~cm}$ ) after withdrawing the maximum velocity of the solid part.

thicknesses of each of these regions tend to reach a steady value for larger distances from the plate tip. Finally, in the last velocity profiles of Fig. 13 (in continuous blue lines) a steady-state regime seems to have been reached and there is no second part of the profile extending at large distance.

These observations are globally consistent with those concerning the deformation field (see Sec. IV). At some distance from the plate tip, the material is essentially deformed elastically, which explains that the velocity profile slowly decreases with distance. Closer to or above the plate tip the velocity profile is expected to be made of one part associated with a liquid flow and one part associated with further deformations in the solid regime.

In order to look at the evolution of the velocity profile in time strictly in the liquid regime we can use the above results (see Sec. IV) concerning the position $(\delta(x)$ ) of the solid-liquid boundary. Between 0 and $\delta$ the material should be liquid, and beyond $(y>\delta(x))$ it should be solid. Let us withdraw, from each velocity profile, the value of the velocity reached at the distance of the solid-liquid limit $(v(\delta))$. This apparent velocity, which as all our PIV measurements in fact corresponds to a displacement over some specific time, results from the deformation of the whole solid region during that time. We can remark (see inset of Fig. 13) a slight evolution of the curvature of the velocity profile toward the apparently steady-state shape associated with the blue lines in Fig. 13. However, it is worth emphasizing that now, except for the two first ones, all the velocity profiles almost fall along the same curve. This means that the velocity profile in the liquid regime is rapidly established around the plate tip, while the apparent variations of the effective velocity are essentially due to residual deformations in the solid regime. Finally, the inset of Fig. 13 provides a direct measurement of the velocity profile inside the liquid BL.

Note that in fact now the maximum velocity is not constant since the velocity induced by deformation in the solid regime has been withdrawn, it progressively increases. The superposition of the velocity profiles remarkably occurs despite this variation, but we have no explanation for that. In addition, it is worth emphasizing that even for the first 
two profiles the slope of the velocity profile along the plate is constant. This means that the shear rate along the plate is almost immediately established, which implies that the shear stress along the plate, given from the constitutive equation in the liquid regime, should rapidly become constant.

\section{B. Flow profile variation as a function of plate velocity}

We measured the steady-state velocity profiles at a large distance from the plate tip (blue region of Fig. 13) for different plate velocities. A first striking result is that the thickness of the flowing region does not change much with velocity: in our range of velocities $\delta$ ranges from 7.5 to $10.3 \mathrm{~mm}$ (see inset of Fig. 14). This result strongly contrasts with the theoretical approaches described in Sec. II which predict that $\delta$ should tend to zero when $V$ tends to zero.

Another striking result is that, if as a first approximate we leave apart this slight increase of $\delta$, over almost three decades of plate velocities the profiles scaled by the plate velocity are almost similar (see Fig. 14). This similarity of the velocity profiles when scaled by the plate velocity suggests that the process by which the BL develops is essentially related to the deformation of the material: indeed, as a first approximate a similar deformation is obtained for a similar displacement of the plate whatever the plate velocity.

Let us now focus on the shape of these velocity profiles. At first sight (from Fig. 14), the shear rate, given by the slope of the profile, does not seem to vary significantly in the flowing region, i.e., from 0 to $\delta$. This could suggest a shear-banding effect analogous to those observed with other materials [Ovarlez et al. (2009)] and associated with a critical shear rate, a rheological parameter of the material. However, since here the velocity profiles are similar if rescaled by the plate velocity the critical shear rate at the liquid-solid transition would widely increase with the plate velocity. This is not consistent with the usual view of shear-banding effect which, as long as the shear-band thickness is much larger than the size of the material elements, predicts a constant critical shear rate at the

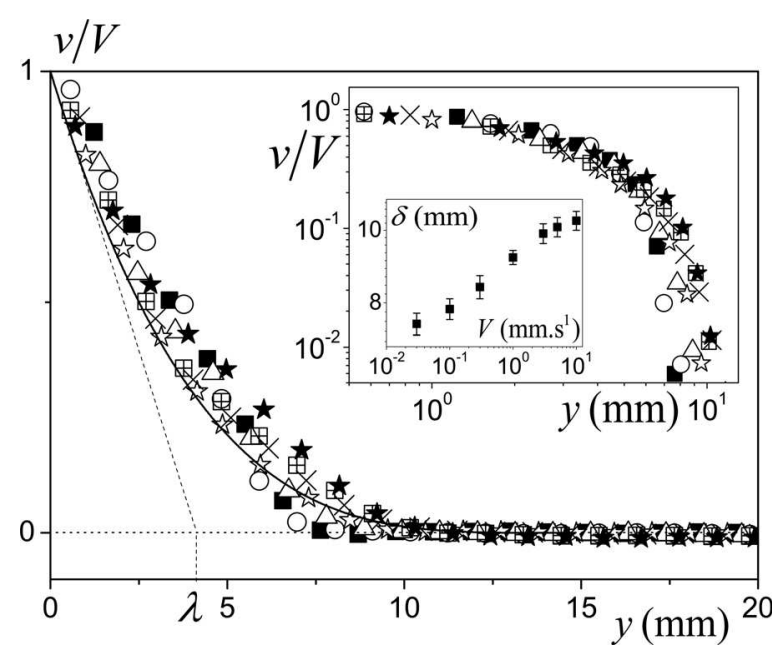

FIG. 14. Scaled velocity profiles at a distance of $15 \mathrm{~cm}$ from the plate tip as a function of the distance from the plate for a Carbopol suspension $(0.5 \%)$ and different plate velocities: 0.03 (squares), 0.1 (open circles), 0.3 (triangles), 1 (stars), 3 (crosses), 5 (cross-squares), $10 \mathrm{~mm} / \mathrm{s}$ (filled stars). The continuous line corresponds to Eq. (7) with $\delta=14.4 \mathrm{~mm}$. The dotted line shows the slope of the velocity profile along the plate for $\lambda=4 \mathrm{~mm}$. Inset: same data in a logarithmic scale, with an inset showing the estimated BL thickness as a function of the velocity. 
interface. Here, $\delta$ is of the order of 500 times the material element size. At last it does not seem either that our data can be explained by the transient shear-banding effects observed with Carbopol gels [Divoux et al. (2010)] in smooth Couette geometries.

Actually a deeper insight in these velocity profiles shows that the shear rate in the liquid region is not constant. We computed directly the apparent shear rate along these profiles from the slope between two successive points and we removed data fluctuating around zero. As expected from the similarity of the scaled velocity profiles we obtained a global similarity of the shear rate profiles, with a clearer difference at the approach of the solid region associated with the slight increase of $\delta$ with the plate velocity (see Fig. 15). The theoretical velocity profile given by Eq. (7) is able to roughly represent the variations of the velocity or the shear rate in the liquid region (see Figs. 14 and 15). However, many other models predicting a progressive decrease of the velocity with the distance and tending to zero when $y \rightarrow \delta$ would also be able to fit the data. The main discrepancy between the model and the data seems to be the fact that in reality the shear rate very slowly decreases over a significant distance from the plate (say, 5-6 mm) and then rapidly decreases to zero (see dotted lines of Fig. 15).

A last, since the scaled velocity profiles very well superimpose close to the plate we can write the shear rate along the plate $V / \lambda$, in which $\lambda$ is a characteristic length of the flow associated with the slope of the velocity profiles along the plate (see Fig. 14). As a consequence, from the simple shear constitutive equation of the material in the liquid regime (3) the shear stress $\tau_{w}$ along the plate is expected to simply write

$$
\tau_{w} / \tau_{c}=1+B i^{-1}
$$

in which $B i^{-1}=\left(k / \tau_{c}\right)(V / \lambda)^{n}$.

\section{FORCE VELOCITY MEASUREMENTS}

We recorded the force $F$ as a function of the depth $h$ of penetration of the plate through the material. Note that due to some surface tension and elasticity effects there is

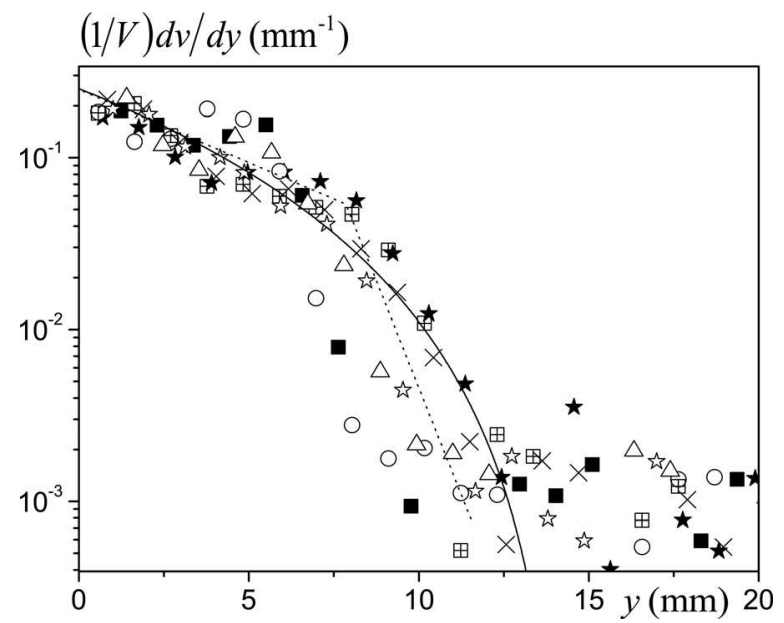

FIG. 15. Local shear rate as a function of the distance from the plate for the data of Fig. 14 with the same symbols. The continuous line corresponds to the model given by Eq. (7) with the parameters of Fig. 14. The dotted lines are guides for the eyes. 
a slight initial deformation of the free surface after the first contact which leads to a shift between the measured and effective values for $h$ but here we will neglect it here. The force vs depth appears to increase almost linearly until the plate is fully immersed then the force remains constant and finally starts to slightly increase when the plate tip approaches the container bottom. This force variation with the immersed depth suggests that the viscous resistance acting on the plate is simply proportional to the immersed depth. As a consequence, except during the first stage of penetration the shear stress $\left(\tau_{w}\right)$ obtained by dividing the force by the surface of contact is almost perfectly constant. Remark that this result is in agreement with our above PIV observation that a uniform flow readily develops behind the leading edge of the plate.

A typical curve of $\tau_{w}$ vs $V$ is shown in Fig. 16. These curves appear to have shapes very similar to the flow curves of yield stress fluids, i.e., with a stress plateau at low shear rates and a slow increase of the stress with increasing shear rate. More precisely the shape of the $\tau_{w}$ vs $V$ curve for a given material appears to be very similar to that of the flow curve of this material obtained from conventional rheometry. It even appears that the flow curve ( $\tau$ vs $\dot{\gamma}$ ) can be approximately obtained by shifting horizontally the $\tau_{w}$ vs $V$ curve by a factor $\lambda$ (see Fig. 16).

The critical stress $\tau_{c}^{\prime}$ estimated from the plateau of these $\tau_{w}$ vs $V$ curves, was shown to be equal to $\tau_{c}$ within $10 \%$, this uncertainty being likely due to the uncertainty on data obtained from rheometry and plate displacement experiments. In the following, in order to strictly focus on the velocity dependence of the stress we compared the data after scaling the stress by the yield stress obtained in each case, which removes the impact of the uncertainty on the latter parameter. We find that when representing the force vs velocity data in terms of $\tau_{w}$ and $V / \lambda$ with a single value for $\lambda$ all the curves fall along (see Fig. 17) the rheometrical flow curves of our Carbopol solutions at different concentrations (which actually are similar when the stress is scaled by the yield stress).

The above result is fully consistent with our observations concerning the shear rate along the plate at different velocities and leading to the stress expression (19). Moreover, the possibility of rescaling the data with the same value for $\lambda$ shows that this parameter does not vary significantly with the yield stress of the fluid (which here was varied from 25 to $60 \mathrm{~Pa}$ ). This suggests that the thickness of the liquid region does not change much either with the yield stress. Note that if we follow the theoretical prediction (7) we should

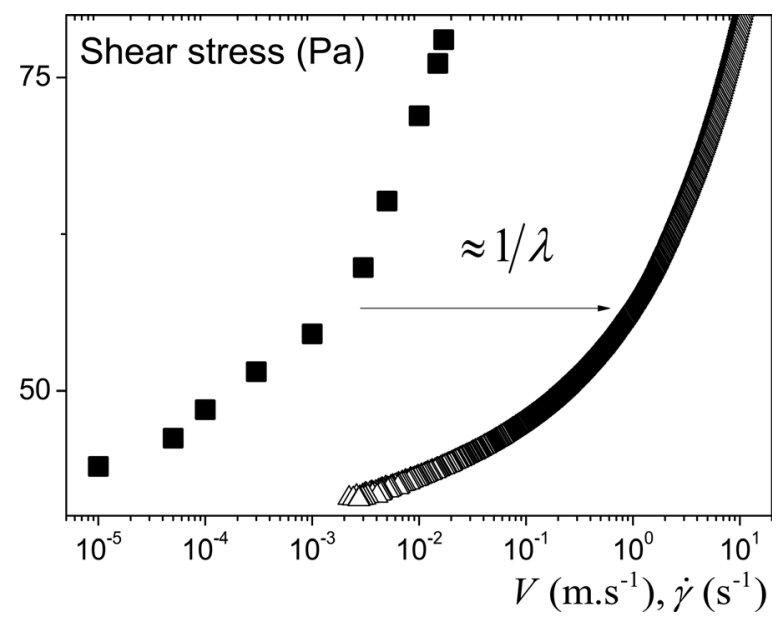

FIG. 16. Carbopol gel $(0.4 \%)$ : flow curve (triangles) and stress vs velocity curve from plate displacement in steady state (squares). 


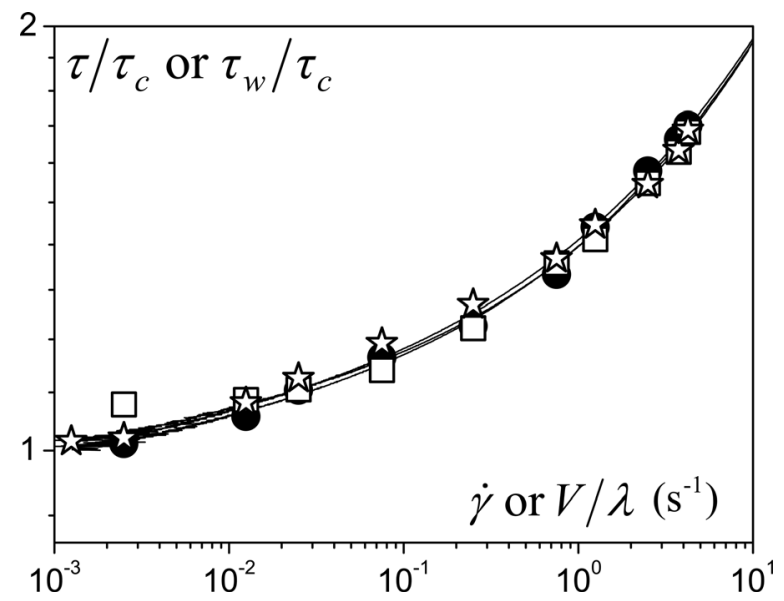

FIG. 17. Flow curves of Carbopol gels (continuous lines) at different concentrations and shear stress as a function of the velocity scaled with $\lambda=4 \mathrm{~mm}$ for the plate displacement data: $0.3 \%$ (squares), $0.4 \%$ (circles), and $0.5 \%$ (stars). In each case, the stress has been scaled by the yielding value determined from the flow curve as described in the text.

have a slight variation of $\lambda$ with the velocity considering that $\delta$ appears to vary (see Sec. V). However, the uncertainly on our force vs velocity and flow curve data do not allow us to reach a definite conclusion on that point: using a constant value for $\lambda$ in our velocity range seems as relevant as trying to vary it slightly.

\section{FINAL DISCUSSION AND CONCLUSION}

In Fig. 18, we propose a summarized description of the process based on all the information obtained in this work. Far ahead of the leading edge of the plate the material is deformed in its solid regime. At a short distance (apparently of the order of $\delta$ ) from the plate tip a solid-liquid transition occurs for the fluid elements along the plate axis, where the deformation is the highest. The thickness of the liquid region (perpendicularly to the plate) then increases rapidly backward along the plate. The BL thickness reaches its steady value in a distance of the order of $\delta$. Thus, the transient characteristics of the BL are essentially situated around the plate tip in a volume of diameter approximately equal to $2 \delta$. Consistently, the stress vs displacement measurement reaches a constant value after a penetration depth of the order of $\delta$. Thus, the thickness of the BL is fixed rapidly after the beginning of the penetration and, in strong contrast with the previous theoretical prediction [cf. Eq. (17)], does not depend at all on the plate length.

The flow characteristics within the uniform BL appear to be similar to the predictions of the basic theory (see Sec. II): the velocity profiles can be reasonably well fitted by Eq. (7) in which one has to adjust the value of $\delta$ (see Fig. 14). There is also a general consistency of the data with the theory through the wall stress expression as a function of the velocity even using a single value of $\delta$ for the different velocities, which is a reasonable assumption considering its slight variation observed by PIV.

Actually one of the most important results of this study is the observation that in our range of data $\delta$ remains larger than a finite value $\left(\delta_{0}\right)$ and very slowly increases with the velocity. This contrast with the natural expectation in line with the previous theoretical prediction [cf. Eq. (17)], that the sheared layer along a solid wall will tend to zero when the relative velocity of the fluid and the wall tend to zero, or more precisely when the shear-dependent term in the stress is much smaller than the yielding term (i.e., large 


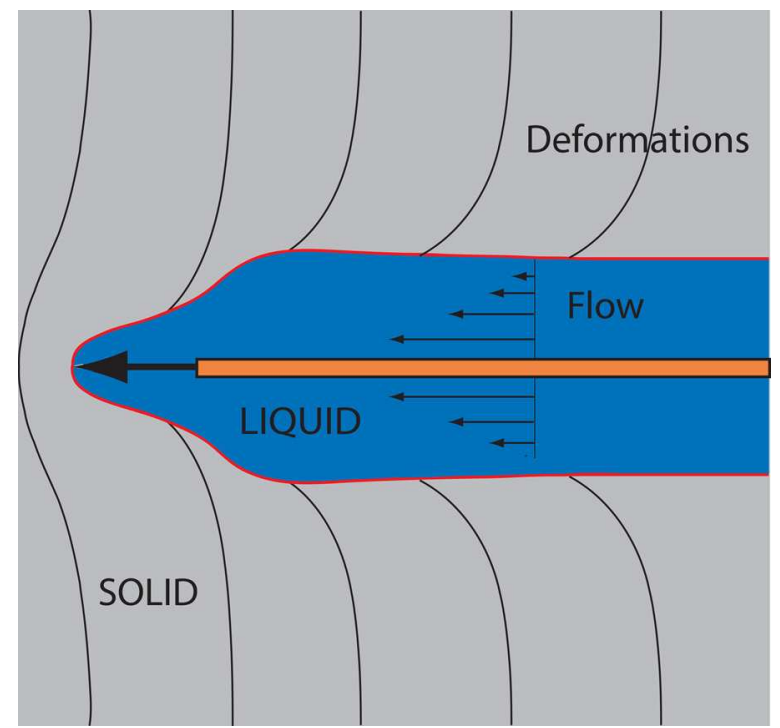

FIG. 18. Schematic aspect of the deformations in the solid region and of the shape of the solid-liquid boundary during the displacement of a plate (orange) through a yield stress fluid (liquid: dark blue, solid: light grey).

Bingham number). It is also remarkable that this thickness cannot easily be linked to a microscopic or macroscopic lengthscale. Nevertheless, it would be useful to be able to predict its value from independent fluid characteristics. Indeed, our data have shown the similarity of the force vs depth curve with the material flow curve. A knowledge of the BL thickness would allow the direct determination of the flow curve of a yield stress fluid from the simple penetration of a plate, an interesting, practical approach for various industrial materials.

A consequence of this minimum finite value for $\delta$ is that according to Eq. (11) $\partial p / \partial x \rightarrow 0$ tends to zero when $V \rightarrow 0$ since $\left(\tau_{w}-\tau_{c}\right) \rightarrow 0$. Such a result completely differs from any flow of a yield stress fluid through a simple geometry. In that case, as for a flow between two fixed plates (see Sec. II), the momentum balance leads to $\partial p / \partial x=\tau_{w} / L$ in which $L$ is a characteristic length of the geometry, which implies that $\partial p / \partial x$ is finite when the velocity tends to zero, i.e., when $\left(\tau_{w}-\tau_{c}\right) \rightarrow 0$. Thus, the liquid region progressively vanishes as the velocity tends to zero. In the present case (flow around a plate), the liquid region keeps an almost constant size whatever the velocity. This means that the BL behaves as if its shape was essentially governed, not by the flow characteristics within this region, but by the conditions around it, namely, the deformations of the solid region and the induced pressure gradient. Remark that this suggestion is consistent with our observations that the velocity profiles scaled by the plate velocity are approximately similar, which led us to conclude that the material deformations are roughly the same after a similar plate displacement at different velocities.

Finally, the general picture we get from these considerations is as follows: when the plate penetrates the material it first deforms it in its solid regime then the liquid region develops where the critical deformation is reached. Under these conditions the deformation field in the solid regime plays a critical role in the determination of liquid region. The thickness of the liquid region is also determined during this period, and the liquid material then flows along the plate behind. This is finally a coupling between the properties in the solid and the liquid regimes which govern the thickness of the BL.

The coupling of solid and liquid behavior at the origin of the BL is likely at the origin of the complete discrepancy of our data with existing theoretical predictions: this 
coupling must be taken into account in the formation of the BL and one cannot simply approach the stress distribution in the solid regime separately. A proper theoretical prediction of the $\mathrm{BL}$ seems to require a treatment taking into account all the aspects of the material behavior. Considering the difficulty of predicting similar processes in solid mechanics as a function of the detailed behavior of the materials [Johnson (1985)], the present task for yield stress fluids appears difficult but interesting as it might provide a way for approaching the behavior of elastoplastic materials under such flow conditions.

More generally our results show that, in contrast with the usual approach so far, the flow characteristics around an object moving through a yield stress significantly depend both on the flow in the liquid region and on the successive deformations of new regions in the solid state. This, for example, likely differs from the situation encountered in a dry granular material [Seguin et al. (2011)] which is unable to store significant elastic energy. In particular, the apparent velocity field around the plate tip finally includes both some deformations in the solid regime and some flow in the liquid regime but measurements relying on successive images of the positions of material elements cannot readily be interpreted in terms of flow in the liquid regime. It is likely that properly modeling this flow type requires a complete modeling of viscous and elastic effects and their couplings at any time such as the approach recently developed by Saramito (2009) and successfully applied to the flow around an obstacle [Cheddadi et al. (2011)].

\section{ACKNOWLEDGMENT}

The authors are grateful to N. Quennouz for her help in the PIV experiments.

\section{References}

Ansley, R. W., and T. N. Smith, "Motion of spherical particles in a Bingham plastic," AIChE J. 13, 1193-1196 (1967).

Atapattu, D. D., R. P. Chhabra, and P. H. T. Uhlherr, "Creeping sphere motion in Herschel-Bulkley fluids: Flow field and drag," J. Non-Newtonian Fluid Mech. 59, 245-265 (1995).

Beaulne, M., and E. Mitsoulis, "Creeping motion of a sphere in tubes filled with Herschel-Bulkley fluids," J. Non-Newtonian Fluid Mech. 72, 55-71 (1997).

Beris, A. N., J. A. Tsamopoulos, R. C. Armstrong, and R. A. Brown, "Creeping motion of a sphere through a Bingham plastic,” J. Fluid Mech. 158, 219-244 (1985).

Blackery, J., and E. Mitsoulis, "Creeping motion of a sphere in tubes filled with a Bingham plastic material," J. Non-Newtonian Fluid Mech. 70, 59-77 (1997).

Cheddadi, I., P. Saramito, B. Dollet, C. Raufaste, and F. Graner, "Understanding and predicting viscous, elastic, plastic flows," Eur. Phys. J. E 34, 1-15 (2011).

Coussot, P., Rheometry of Pastes, Suspensions and Granular Materials (Wiley, New York, 2005).

Coussot, P., and G. Ovarlez, “A physical approach to shear-banding of jammed systems," Eur. Phys. J. E 33, 183-188 (2010).

Coussot, P., H. Tabuteau, X. Chateau, L. Tocquer, and G. Ovarlez, "Aging and solid or liquid behavior in pastes," J. Rheol. 50, 975-994 (2006).

Coussot, P., L. Tocquer, C. Lanos, and G. Ovarlez, "Macroscopic vs local rheology of yield stress fluids," J. Non-Newtonian Fluid Mech. 158, 85-90 (2009).

Divoux, T., D. Tamarii, C. Barentin, and S. Manneville, "Transient shear banding in a simple yield stress fluid," Phys. Rev. Lett. 104, 208301 (2010).

Dollet, B., and F. Graner, "Two-dimensional flow of foam around a circular obstacle: Local measurements of elasticity, plasticity and flow,” J. Fluid Mech. 585, 181-211 (2007).

Goyon, J., A. Colin, and L. Bocquet, "How does a soft glassy material flow: Finite size effects, non local rheology and flow cooperativity," Soft Matter 6, 2668-2678 (2010). 
Gueslin, B., L. Talini, and Y. Peysson, "Sphere settling in an aging yield stress fluid: Link between the induced flows and the rheological behavior," Rheol. Acta 48, 961-970 (2009).

Habdas, P., D. Schaar, A. C. Levitt, and E. R. Weeks, "Forced motion of a probe particle near the colloidal glass transition," Europhys. Lett. 67, 477-483 (2004).

Johnson, K. L., Contact Mechanics (Cambridge University Press, Cambridge, 1985).

Jossic, L., and A. Magnin, "Drag and stability of objects in a yield stress fluid," AIChE J. 47, 2666-2672 (2001).

Lootens, D., P. Jousset, L. Martinie, N. Roussel, and R. J. Flatt, "Yield stress during setting of cement pastes from penetration tests," Cem. Concr. Res. 39, 401-408 (2009).

Lowery, B., and J. E. Morrison, "Soil penetrometers and penetrability," in Methods of Soil Analysis-Part 4 Physical Methods, edited by J. H. Dane and G. C. Topp (Soil Science Society of America Book Series: 5, Madison, WI, 2002), pp. 363-368.

Merkak, O., L. Jossic, and A. Magnin, "Spheres and interactions between spheres moving at low velocities in a yield stress fluid," J. Non-Newt. Fluid Mech. 133, 99-108 (2006).

Oldroyd, J. D., "Two-dimensional plastic flow of a Bingham solid-A plastic boundary-layer theory for slow motion," Math. Proc. Cambridge Philos. Soc. 43, 383-395 (1947).

Oppong, F., and J. R. de Bruyn, "Mircorheology and jamming in a yield-stress fluid,” Rheol. Acta 50, 317-326 (2011).

Oppong, F., P. Coussot, and J. R. de Bruyn, "Gelation on the microscopic scale," Phys. Rev. E 78, 021405 (2008).

Ovarlez, G., K. Krishan, and S. Cohen-Addad, "Investigation of shear banding in three-dimensional foams," Europhys. Lett. 91, 68005 (2010).

Ovarlez, G., S. Rodts, A. Ragouilliaux, P. Coussot, and J. Goyon, "Wide-gap Couette flows of dense emulsions: Local concentration measurements, and comparison between macroscopic and local constitutive law measurements through magnetic resonance imaging," Phys. Rev. E 78, 036307 (2008).

Ovarlez, G., S. Rodts, X. Chateau, and P. Coussot, "Phenomenology and physical origin of shear-localization and shear-banding in complex fluids," Rheol. Acta 48, 831-844 (2009).

Piau, J. M., "Carbopol gels: Elastoviscoplastic and slippery glasses made of individual swollen sponges mesoand macroscopic properties, constitutive equations and scaling laws," J. Non-Newtonian Fluid Mech. 144, 1-29 (2007).

Piau, J. M., "Viscoplastic boundary layer,” J. Non-Newtonian Fluid Mech. 102, 193-218 (2002).

Piau, J. M., and K. Debiane, "The adhesive or slippery flat plate viscoplastic boundary layer for a shear-thinning power-law viscosity,” J. Non-Newtonian Fluid Mech. 117, 97-107 (2004).

Putz, A., and I. A. Frigaard, "Creeping flow around particles in a Bingham fluid," J. Non-Newtonian Fluid Mech. 165, 263-280 (2010).

Putz, A. M. V., T. I. Burghelea, I. A. Frigaard, and D. M. Martinez, "Settling of an isolated spherical particle in a yield stress shear thinning fluid," Phys. Fluids 20, 033102 (2008).

Ragouilliaux, A., G. Ovarlez, N. Shahidzadeh-Bonn, B. Herzhaft, T. Palermo, and P. Coussot, "Transition from a simple yield stress fluid to a thixotropic material," Phys. Rev. E 76, 051408 (2007).

Rabideau, B. D., P. Moucheront, F. Bertrand, S. Rodts, N. Roussel, C. Lanos, and P. Coussot, "The extrusion of a model yield stress fluid imaged by MRI velocimetry," J. Non-Newtonian Fluid Mech. 165, 394-408 (2010).

Saramito, P., "A new elastoviscoplastic model based on the Herschel-Bulkley viscoplastic model," J. NonNewtonian Fluid Mech. 158, 154-161 (2009).

Schlichting, H., Boundary Layer Theory (McGraw-Hill, New York, 1968).

Seguin, A., Y. Bertho, P. Gondret, and J. Crassous, "Dense granular flow around a penetrating object: Experiment and hydrodynamic model,” Phys. Rev. Lett. 107, 048001 (2011).

Tabuteau, H., P. Coussot, and J. de Bruyn, "Drag force on a sphere in steady motion through a yield stress fluid,” J. Rheol. 51, 125-137 (2007).

Tokpavi, D. L., A. Magnin, and P. Jay, "Very slow flow of Bingham viscoplastic fluid around a circular cylinder," J. Non-Newtonian Fluid Mech. 154, 65-76 (2008).

Tokpavi, D. L., P. Jay, A. Magnin, and L. Jossic, "Experimental study of the very slow flow of a yield stress fluid around a circular cylinder,” J. Non-Newtonian Fluid Mech. 164, 35-44 (2009).

Uhlherr, P. H. T., J. Guo, T. N. Fang, and C. Tiu, "Static measurement of yield stress using a cylindrical penetrometer," Korea-Aust. Rheol. J. 14, 17-23 (2002).

White, F. M., Viscous Fluid Flow (McGraw-Hill, New York, 1991). 